\title{
CHARACTERIZATIONS OF COLLECTIVELY PRECOMPACT AND SEMI-PRECOMPACT OPERATORS ON TOPOLOGICAL VECTOR SPACES
}

\author{
M. V. DESHPANDE and S. M. PADHYE
}

(Received 27 July 1978)

Communicated by Gavin Brown

\begin{abstract}
Characterizations of collectively precompact and collectively semi-precompact sets of operators on topological vector spaces are obtained. These lead to the characterization of totally bounded sets of semi-precompact operators on locally convex spaces.
\end{abstract}

1980 Mathematics subject classification (Amer. Math. Soc.): primary 47 B 05, 47 D 15; secondary 46 A 05, 46 A 15.

\section{Introduction}

Let $X, Y$ be topological vector spaces and $L_{b}(X, Y)$ the space of all continuous linear operators on $X$ into $Y$ equipped with topology of uniform convergence on bounded sets. A subset $\mathscr{K}$ of $L_{b}(X, Y)$ is said to be collectively precompact if there exists a 0 -neighbourhood $V$ in $X$ such that $\mathscr{K}(V)=\{K(x): K \in \mathscr{K}, x \in V\}$ is totally bounded in $Y$. Characterizations of collectively precompact sets were obtained by Palmer (1969) and DePree and Klein (1974), where $X, Y$ are normed linear spaces. In the characterizations obtained by them, the spaces $X, Y$ appear explicitly in a natural way. But since collectively precompact sets are subsets of $L_{b}(X, Y)$, it is equally natural to think of some characterization which is intrinsic to $L_{b}(X, Y)$ in the sense that it makes reference only to $L_{b}(X, Y)$. In this paper, a characterization of this type is obtained. It also sheds some light on other properties of collectively precompact sets which follow easily. A characterization of totally bounded sets of precompact operators in normed spaces was obtained by Palmer (1969). This was extended by Geue (1974) to infra-barrelled locally convex spaces. It is proved in this paper that the condition infra-barrelled is not necessary. 
Before we prove our results, it will be convenient to go through notations and preliminaries. Let $X, Y$ denote topological vector spaces. We denote by $L(X, Y)$ the space of all continuous linear operators from $X$ to $Y . L_{b}(X, Y)$ will denote the space $L(X, Y)$ equipped with the topology of uniform convergence on bounded sets of $X$. The typical neighbourhood of 0 in $L_{b}(X, Y)$ will be denoted by $N(A, U)=\{T \in L(X, Y): T(A) \subset U\}$, where $A$ is a bounded subset of $X$ and $U$ a 0 -neighbourhood in $Y$. Let $X^{*}, Y^{*}$ denote the topological duals of $X, Y$ respectively. and $X_{s}^{*}, Y_{s}^{*}$ the spaces $X^{*}, Y^{*}$ with strong topologies. For $x_{0}$ in $X$ and $x_{0}^{*}$ in $X^{*}$, we define the operator $x_{0} \otimes x_{0}^{*}$ on $X$ by the equation

$$
\left(x_{0} \otimes x_{0}^{*}\right)(x)=x_{0}^{*}(x) x_{0} .
$$

Similarly, if $y_{0} \in Y$ and $x_{0}^{*} \in X^{*}$, then we define the operator $y_{0} \otimes x_{0}^{*}$ from $X$ into $Y$ by $\left(y_{0} \otimes x_{0}^{*}\right)(x)=x_{0}^{*}(x) y_{0}$. The operator $y_{0} \otimes y_{0}^{*}$ on $Y$ is similarly defined, where $y_{0}^{*} \in Y^{*}$. When $X=Y$, we write $L_{b}(X, Y)=L_{b}(X)$.

Definition. Let $X, Y$ be topological vector spaces and $\mathscr{K}$ a subset of $L(X, Y)$. $\mathscr{K}$ is collectively precompact if there exists a 0 -neighbourhood $V$ in $X$ such that $\mathscr{K}(V)$ is totally bounded in $Y . \mathscr{K}$ is collectively semi-precompact if $\mathscr{K}(B)$ is totally bounded in $Y$ for every bounded set $B$ in $X$.

In this section, we shall obtain characterizations of collectively precompact and semi-precompact operators intrinsic to $L_{b}(X, Y)$ as described in the Introduction.

Proposition 1.1. Let $X, Y$ be topological vector spaces such that $X$ has nontrivial topological dual. Let $E=x_{0} \otimes x_{0}^{*}, \quad x_{0}^{*}\left(x_{0}\right)=1, \quad x_{0} \in X, x_{0}^{*} \in X^{*}$. Then for $\mathscr{K} \subset L(X, Y)$ the following are equivalent:

(i) $\mathscr{K}$ is collectively precompact.

(ii) $\mathscr{K} W E$ is totally bounded subset of $L_{b}(X, Y)$ for some 0 -neighbourhood $W$ in $L_{b}(X)$.

Proof. Assume (i). Let $U$ be a balanced neighbourhood of 0 in $X$ such that $\mathscr{K}(U)$ is totally bounded in $Y$. Then $W=N\left(x_{0}, U\right)$ is a 0 -neighbourhood in $L_{b}(X)$. Obviously, $W\left(x_{0}\right)=\left\{T\left(x_{0}\right): T \in W\right\} \subset U$. Hence $\mathscr{K} W\left(x_{0}\right) \subset \mathscr{K}(U)$ is totally bounded. Now to prove the total boundedness of $\mathscr{K} W E$, let $N(B, V)$ be any 0 neighbourhood in $L_{b}(X, Y)$. We may assume that $V$ is balanced. Choose $k>\sup \left\{\left|x_{0}^{*}(x)\right|: x \in B\right\}$. Since $\mathscr{K} W\left(x_{0}\right)$ is totally bounded, there exist

$$
K_{1}, K_{2}, \ldots, K_{m} \text { in } \mathscr{K} \text { and } T_{1}, T_{2}, \ldots, T_{m} \text { in } W
$$


such that for every $K$ in $\mathscr{K}$ and $T$ in $W$, there are $K_{i}, T_{i}$ with

$$
K T\left(x_{0}\right)-K_{i} T_{i}\left(x_{0}\right) \in V / k .
$$

Then for any $x$ in $B$, we have

$$
\left(K T E-K_{i} T_{i} E\right)(x)=x_{0}^{*}(x)\left[K T\left(x_{0}\right)-K_{i} T_{i}\left(x_{0}\right)\right] \in\left[x_{0}^{*}(x) / k\right] V
$$

$$
\subset\left[\left|x_{0}^{*}(x)\right| / k\right] V \subset V .
$$

Thus $\left(K T E-K_{i} T_{i} E\right)(B) \subset V$. Equivalently, $K T E-K_{i} T_{i} E \in N(B, V)$. This proves the total boundedness of $\mathscr{K} W E$ in $L_{b}(X, Y)$ which is (ii).

Assume (ii). Let $W=N(A, U)$ be neighbourhood of 0 in $L_{b}(X)$ such that $\mathscr{K} W E$ is totally bounded in $L_{b}(X, Y)$. We may assume $U$ to be balanced. Let

$$
k>\sup \left\{\left|x_{0}^{*}(x)\right|: x \in A\right\}
$$

and put $V=U / k$. Then for $v$ in $V$ and $x$ in $A$, we have

$$
\left(v \otimes x_{0}^{*}\right)(x)=x_{0}^{*}(x) v \in\left|x_{0}^{*}(x)\right| V \subset k V=U .
$$

It follows that $v \otimes x_{0}^{*} \in N(A, U)$ for each $v$ in $V$. Since

$$
v=\left(v \otimes x_{0}^{*}\right)\left(x_{0}\right) \in N(A, U)\left(x_{0}\right), V \subset N(A, U)\left(x_{0}\right) .
$$

But $\mathscr{K} N(A, U)\left(x_{0}\right)$ is totally bounded in $Y$ by the assumption on $\mathscr{K} W E$. Hence $\mathscr{K}(V)$ is totally bounded in $Y$ which proves (i).

By adapting the arguments of the above proof, the following corollaries can be easily deduced.

COROLLARY 1.2. If $\mathscr{K} W E$ is totally bounded in the pointwise topology, then it is so in $L_{b}(X, Y)$.

COROLlaRY 1.3. $\mathscr{K}$ is totally bounded in the pointwise topology if and only if $\mathscr{K} S E$ is totally bounded in $L_{b}(X, Y)$ for every $S$ in $L(X)$.

The following proposition constitutes a generalization of Theorem 2.10 of DePree and Klein (1974) to locally convex spaces. For that, we first prove

Lemma 1.4. Let $X, Y$ and $Z$ be topological vector spaces such that $Y$ is barrelled and $Z$ locally convex. Let $\mathscr{K} \subset L(X, Y), \mathscr{F} \subset L(Y, Z)$ be such that both are pointwise totally bounded. Then $\mathscr{F} \mathscr{K} \subset L(X, Z)$ is also pointwise totally bounded.

Proof. Since $\mathscr{F}$ is pointwise totally bounded, it is pointwise bounded and barrelledness of $Y$ implies that $\mathscr{F}$ is equicontinuous. To prove the pointwise total boundedness of $\mathscr{F} \mathscr{K}$, let $x_{1} \in X$ and $U$ any 0 -neighbourhood in $Z$. Then there exists a 0 -neighbourhood $V$ in $Y$ such that $\mathscr{F}(V) \subset U$. Since $\mathscr{K}$ is pointwise 
totally bounded, $\mathscr{K}\left(x_{1}\right)$ is totally bounded in $Y$. Hence there exist $K_{1}, K_{2}, \ldots, K_{m}$ in $\mathscr{K}$ such that for every $K$ in $\mathscr{K}$, there is $K_{i}$ with $K\left(x_{1}\right)-K_{i}\left(x_{1}\right) \in V$. Since $\mathscr{F}$ is pointwise totally bounded, $\bigcup\left\{\mathscr{F} K_{i}\left(x_{1}\right): i=1,2, \ldots, m\right\}$ is totally bounded. Now it is enough to show that $\bigcup\left\{\mathscr{F} K_{i}\left(x_{1}\right): i=1,2, \ldots, m\right\}$ is a $U$-net for $\mathscr{F} \mathscr{K}\left(x_{1}\right)$. Let $F \in \mathscr{F}$ and $K \in \mathscr{K}$. Then for some $i, K\left(x_{1}\right)-K_{i}\left(x_{1}\right) \in V$. Hence

$$
F K\left(x_{1}\right)-F K_{i}\left(x_{1}\right) \in F(V) \subset U
$$

which proves the required result.

Proposition 1.5. Let $X, Y$ and $Z$ be topological vector spaces such that $X$ has nontrivial dual, $Y$ is barrelled and $Z$ is locally convex. Let $\mathscr{K} \subset L(X, Y)$ be collectively precompact. For $\mathscr{F} \subset L_{b}(Y, Z)$, suppose that $\mathscr{F}(y)$ is totally bounded for each $y$ in $Y$. Then $\mathscr{F} \mathscr{K}=\{F K: F \in \mathscr{F}, K \in \mathscr{K}\}$ is collectively precompact.

ProOF. Let $E=x_{0} \otimes x_{0}^{*}, x_{0}^{*}\left(x_{0}\right)=1, x_{0} \in X, x_{0}^{*} \in X^{*}$. Since $\mathscr{K}$ is collectively precompact by Proposition 1.1, there is $W=N(A, U)$ a 0-neighbourhood in $L_{b}(X)$ such that $\mathscr{K} W E$ is totally bounded in $L_{b}(X, Y)$. Hence $\mathscr{K} W E$ is pointwise totally bounded. By Lemma 1.4, we get the pointwise total boundedness of $\mathscr{F} \mathscr{K} W E$ in $L(X, Z)$. Now by Corollary $1.2, \mathscr{F} \mathscr{K} W E$ is also totally bounded in $L_{b}(X, Z)$. Proposition 1.1 now gives collective precompactness of $\mathscr{F} \mathscr{K}$.

Proposition 1.6. Suppose $X, Y$ are topological vector spaces with nontrivial duals $X^{*}, Y^{*}$. Let $F=y_{0} \otimes y_{0}^{*}, \quad y_{0}^{*}\left(y_{0}\right)=1, \quad y_{0} \in Y$ and $y_{0}^{*} \in Y^{*}$. Suppose $\mathscr{K} \subset L(X, Y)$ and $\mathscr{K}^{*}$ the dual family. Then the following are equivalent:

(i) $\mathscr{K}^{*} \subset L\left(Y_{s}^{*}, X_{s}^{*}\right)$ is collectively precompact.

(ii) $F W \mathscr{K}$ is totally bounded for some 0-neighbourhood $W$ in $L_{b}(Y)$.

Proof. Assume (i). Then $\mathscr{K}^{*}\left(B^{0}\right)$ is totally bounded in $X_{s}^{*}$, where $B$ is some bounded set in $Y$ and $B^{0}$ its polar in $Y^{*}$. Let $U=\left\{y:\left|y_{0}^{*}(y)\right| \leqslant 1\right\}$. Then $y_{0} \in U$ and $U$ is a 0 -neighbourhood in $Y$. First we prove that if $W=N(B, U)$, then $W^{*}\left(y_{0}^{*}\right) \subset B^{0}$. For this, let $y^{*} \in W^{*}\left(y_{0}^{*}\right)$. Then $y^{*}=T^{*}\left(y_{0}^{*}\right)$ for some $T$ in $N(B, U)$. Therefore, $T(B) \subset U$ which implies that $[T(B)]^{0} \supset U^{0}$. Since the latter set contains $y_{0}^{*}, y_{0}^{*} \in[T(B)]^{0}$. This means that $T^{*}\left(y_{0}^{*}\right) \in B^{0}$ and hence $W^{*}\left(y_{0}^{*}\right) \subset B^{0}$. Therefore $\mathscr{K}^{*} W^{*}\left(y_{0}^{*}\right) \subset \mathscr{K}^{*}\left(B^{0}\right)$ is totally bounded in $X_{s}^{*}$. Now to show the total boundedness of $F W \mathscr{K}$ in $L_{b}(X, Y)$, let $N(A, V)$ be any 0-neighbourhood in $L_{b}(X, Y)$. Let $\alpha_{0}>0$ be such that $|\alpha| \leqslant \alpha_{0}$ implies $\alpha y_{0} \in V$. Since $\mathscr{K}^{*} W^{*}\left(y_{0}^{*}\right)$ is totally bounded in $X_{s}^{*}$, it follows that for $V^{*}=\left\{x^{*} \in X^{*}:\left|x^{*}(x)\right| \leqslant \alpha_{0}, x \in A\right\}$, a 0-neighbourhood in $X_{s}^{*}$, there exist $K_{1}, K_{2}, \ldots, K_{m}$ in $\mathscr{K}$ and $T_{1}, T_{2}, \ldots, T_{m}$ in $W$ such that for every $K$ in $\mathscr{K}$ and $T$ in $W$, there are $K_{i}, T_{i}$ with $\left|\left[K^{*} T^{*}\left(y_{0}^{*}\right)-K_{i}^{*} T_{i}^{*}\left(y_{0}^{*}\right)\right](x)\right| \leqslant \alpha_{0}$ for all $x$ in $A$. Equivalently, $\left|y_{0}^{*}\left(T K-T_{i} K_{i}\right)(x)\right| \leqslant \alpha_{0}$ for all $x$ in $A$. Hence for any $x$ in $A$, we have $\left(F T K-F T_{i} K_{i}\right)(x)=y_{0}^{*}\left[\left(T K-T_{i} K_{i}\right)(x)\right] y_{0}=\alpha y_{0}$, where 
$|\alpha| \leqslant \alpha_{0}$. Thus $\left.\left(F T K-F T_{i} K_{i}\right)\right)(x) \in V$ for all $x$ in $A$. Equivalently,

$$
F T K-F T_{i} K_{i} \in N(A, V) \text {. }
$$

This proves (ii).

Assume (ii). Let $W=N(B, U)$ be the 0-neighbourhood in $L_{b}(Y)$ such that $F W \mathscr{K}$ is totally bounded in $L_{b}(X, Y)$. First we show that $y_{0}^{*} W \mathscr{K}$ is totally bounded in $X_{s}^{*}$. Let $\beta>0$ and $A$ be any bounded subset of $X$. Then $V=\left\{y:\left|y_{0}^{*}(y)\right| \leqslant \beta\right\}$ is a 0-neighbourhood in $Y$. By total boundedness of $F W \mathscr{K}$, it follows that for $N(A, V)$, there exist $K_{1}, K_{2}, \ldots, K_{m}$ in $\mathscr{K}$ and $T_{1}, T_{2}, \ldots, T_{m}$ in $W$ such that for every $K$ in $\mathscr{K}$ and $T$ in $W$, there are $K_{i}, T_{i}$ with $F\left(T K-T_{i} K_{i}\right)(A) \subset V$. Equivalently,

$$
\left|y_{0}^{*}\left[y_{0}^{*}\left(T K-T_{i} K_{i}\right)(x) y_{0}\right]\right| \leqslant \beta \text { for all } x \text { in } A .
$$

$y_{0}^{*}\left(y_{0}\right)=1$ gives

$$
\left|y_{0}^{*}\left(T K-T_{i} K_{i}\right)(x)\right| \leqslant \beta \text { for all } x \text { in } A .
$$

This proves the total boundedness of $y_{0}^{*} W \mathscr{K}=\mathscr{K}^{*} W^{*}\left(y_{0}^{*}\right)$ in $X_{s}^{*}$. Let $\alpha_{0}>0$ be such that $|\alpha| \leqslant \alpha_{0}$ implies $\alpha y_{0} \in U$. Now to prove (i), it is enough to show that $B^{0} \subset\left(1 / \alpha_{0}\right) W^{*}\left(y_{0}^{*}\right)$. Let $y^{*} \in B^{0}$ and $T=\alpha_{0}\left(y_{0} \otimes y^{*}\right)$. Then $y^{*}=\left(1 / \alpha_{0}\right) T^{*}\left(y_{0}^{*}\right)$. Also $\left|y^{*}(y)\right| \leqslant 1$ for all $y$ in $B$ and hence $\alpha_{0} y^{*}(y) y_{0} \in U$. Thus

$$
T(y)=\alpha_{0} y^{*}(y) y_{0} \in U \text { for all } y \text { in } B
$$

and we have $T \in N(B, U)=W$. This proves that $B^{0} \subset\left(1 / \alpha_{0}\right) W^{*}\left(y_{0}^{*}\right)$, and hence (i) is proved.

The technique of the above proof can be used to deduce the following corollary, the proof of which is omitted.

COROLlaRY 1.7. $\mathscr{K}^{*}$ is totally bounded in the pointwise topology if and only if FS $\mathscr{K}$ is totally bounded in $L_{b}(X, Y)$ for every $S$ in $L(Y)$.

Now we prove the similar characterization for $\mathscr{K}$ and $\mathscr{K}^{*}$ to be collective semiprecompact.

Proposition 1.8. Let $X, Y$ be topological vector spaces such that $X$ has nontrivial topological dual. Let $E=x_{0} \otimes x_{0}^{*}, x_{0}^{*}\left(x_{0}\right)=1, x_{0} \in X, x_{0}^{*} \in X^{*}$ and $\mathscr{K} \subset L(X, Y)$. Then the following are equivalent:

(i) $\mathscr{K}$ is collectively semi-precompact.

(ii) $\mathscr{K} W E$ is totally bounded in $L_{b}(X, Y)$ for every bounded set $W$ of $L_{b}(X)$.

Proof. Assume (i). Let $W$ be any bounded set in $L_{b}(X)$. To prove the total boundedness of $\mathscr{K} W E$ in $L_{b}(X, Y)$, let $N(A, V)$ be a 0 -neighbourhood in $L_{b}(X, Y)$. 
Let $k>\sup \left\{\left|x_{0}^{*}(x)\right|: x \in A\right\}$. Since $W$ is bounded, $W\left(x_{0}\right)$ is bounded in $X$ and therefore by the assumption $\mathscr{K} W\left(x_{0}\right)$ is totally bounded in $Y$. Hence there exist $K_{1}, K_{2}, \ldots, K_{p}$ in $\mathscr{K}$ and $T_{1}, T_{2}, \ldots, T_{p}$ in $W$ such that for every $K$ in $\mathscr{K}$ and $T$ in $W$, there are $T_{j}, K_{j}$ with $K T\left(x_{0}\right)-K_{j} T_{j}\left(x_{0}\right) \in V / k$. Then for any $x$ in $A$, we have

$$
\left(K T E-K_{j} T_{j} E\right)(x)=x_{0}^{*}(x)\left[K T\left(x_{0}\right)-K_{j} T_{j}\left(x_{0}\right)\right] \in x_{0}^{*}(x)(V / k) \subset V .
$$

Hence $K T E-K_{j} T_{j} E \in N(A, V)$. This proves (ii).

Assume (ii). Let $A$ be any bounded subset of $X$. Suppose that

$$
W=\left\{x \otimes x_{0}^{*}: x \in A\right\} \text {. }
$$

Then obviously, $W\left(x_{0}\right)=A$. Also for any bounded set $B$ in $X$, we have

$$
W(B)=\left\{\left(x \otimes x_{0}^{*}\right)(t): x \in A, t \in B\right\}=x_{0}^{*}(B) A
$$

which is bounded in $X$. Hence, $W$ is bounded in $L_{b}(X)$. Therefore, by assumption, $\mathscr{K} W E$ is totally bounded in $L_{b}(X, Y)$. It follows that

$$
\mathscr{K}(A)=\mathscr{K} W\left(x_{0}\right)=\mathscr{K} W E\left(x_{0}\right)
$$

is also totally bounded in $Y$. This shows (i).

Proposition 1.9. Let $X, Y$ be topological vector spaces with nontrivial topological duals where $Y$ is Hausdorff. Let $F=y_{0} \otimes y_{0}^{*}, y_{0}^{*}\left(y_{0}\right)=1, y_{0} \in Y, y_{0}^{*} \in Y^{*}$ and $\mathscr{K} \subset L(X, Y)$. Then the following are equivalent:

(i) $\mathscr{K}^{*} \subset L\left(Y_{s}^{*}, X_{s}^{*}\right)$ is collectively semi-precompact.

(ii) $F W \mathscr{K}$ is totally bounded in $L_{b}(X, Y)$ for every bounded set $W$ in $L_{b}(Y)$.

Proor. Suppose (i) is true and $W$ is any bounded subset of $L_{b}(Y)$. Let $N(A, V)$ be any arbitrary neighbourhood of 0 in $L_{b}(X, Y)$. Choose $\alpha_{0}>0$ so that $|\alpha| \leqslant \alpha_{0}$ implies $\alpha y_{0} \in V$. Since $W$ is bounded in $L_{b}(Y), W^{*}\left(y_{0}^{*}\right)=y_{0}^{*} W$ is bounded in $Y_{s}^{*}$ and therefore, by assumption, $\mathscr{K}^{*} W^{*}\left(y_{0}^{*}\right)$ is totally bounded in $X_{s}^{*}$. Hence there exist $K_{1}, K_{2}, \ldots, K_{p}$ in $\mathscr{K}$ and $T_{1}, T_{2}, \ldots, T_{p}$ in $W$ such that for each $K$ in $\mathscr{K}$ and $T$ in $W$, there are $K_{j}, T_{j}$ satisfying $\left|\left(K^{*} T^{*}-K_{j}^{*} T_{j}^{*}\right)\left(y_{0}^{*}\right)(x)\right| \leqslant \alpha_{0}$ for all $x$ in $A$. Equivalently, $\left|y_{0}^{*}\left[\left(T K-T_{j} K_{j}\right)(x)\right]\right| \leqslant \alpha_{0}$ for each $x$ in $A$. Hence

$$
y_{0}^{*}\left[\left(T K-T_{j} K_{j}\right)(x)\right] y_{0} \in V \text { for all } x \text { in } A .
$$

But this means that $F\left(T K-T_{j} K_{j}\right)(A) \subset V$ which shows (ii).

Assume (ii). Let $B^{*}$ be any bounded subset of $Y_{s}^{*}$. Suppose

$$
W=\left\{y_{0} \otimes y^{*}: y^{*} \in B^{*}\right\} \text {. }
$$

It is easy to verify that $W$ is bounded in $L_{b}(Y)$ and that $W^{*}\left(y_{0}^{*}\right)=B^{*}$. Now by Hausdorff property of $Y$, we can choose a balanced 0-neighbourhood $V$ in $Y$ such that $y_{0} \notin V$. Let $p$ denote the Minkowski functional of $V$. Then $p\left(y_{0}\right) \neq 0$ and $p(\alpha y)=|\alpha| p(y)$ for all $y \in Y$. Let $V^{*}=\left\{x^{*} \in X^{*}:\left|x^{*}(x)\right| \leqslant \beta, x \in A\right\}, \beta>0$, 
be any 0-neighbourhood in $X_{s}^{*}$. Since $F W \mathscr{K}$ is totally bounded in $L_{b}(X, Y)$ for the neighbourhood of $0, N\left(\left(1 / \beta p\left(y_{0}\right)\right) A, V\right)$ in $L_{b}(X, Y)$, there exist $K_{1}, K_{2}, \ldots, K_{m}$ in $\mathscr{K}$ and $T_{1}, T_{2}, \ldots, T_{m}$ in $W$ such that for every $K$ in $\mathscr{K}$ and $T$ in $W$, there are $K_{i}, T_{i}$ with $\left[1 / \beta p\left(y_{0}\right)\right]\left(F T K-F T_{i} K_{i}\right)(A) \subset V$. Hence we must have

$$
\left[1 / \beta p\left(y_{0}\right)\right] p\left[\left(F T K-F T_{i} K_{i}\right)(x)\right] \leqslant 1 \text { for all } x \text { in } A .
$$

Simplifying we get

$$
p\left[\left(F T K-F T_{i} K_{i}\right)(x)\right]=\left|y_{0}^{*}\left[\left(T K-T_{i} K_{i}\right)(x)\right]\right| p\left(y_{0}\right) \leqslant \beta p\left(y_{0}\right) \text { for all } x \text { in } A .
$$

Thus

$$
\left|y_{0}^{*}\left[\left(T K-T_{i} K_{i}\right)(x)\right]\right| \leqslant \beta \text { for all } x \text { in } A .
$$

This shows that $y_{0}^{*}\left(T K-T_{i} K_{i}\right) \in V^{*}$. Hence, $y_{0}^{*} W \mathscr{K}=\mathscr{K}^{*} W^{*}\left(y_{0}^{*}\right)=\mathscr{K}^{*}\left(B^{*}\right)$ is totally bounded in $X_{s}^{*}$ which proves (i).

The characterizations obtained in Section 1 will now be used to generalize Theorem 2.1 of Palmer (1969) to locally convex Hausdorff spaces. A generalization of Palmer's result was obtained by Geue (1974) for infra-barrelled spaces. However, it is proved here that such an assumption is not necessary. The proof will depend upon the following lemma, the proof of which is omitted because it is a straightforward generalization of a similar result in metric spaces (see Anselone (1970)).

For this lemma, the concept of smallness of order $U$ is required, where $U$ is a 0-neighbourhood in a topological vector space.

Definition. A subset $S$ of a topological vector space is said to be small of order $U$ if for all points $x, y \in S$ we have $x-y \in U$.

LEMMA 2.1. A subset $S$ of a topological vector space is totally bounded if and only if for each neighbourhood $U$ of 0 and each infinite set $T \subset S$, there is an infinite set $T^{\prime} \subset T$ small of order $U$.

THEOREM 2.2. Let $X, Y$ be locally convex Hausdorff spaces and $\mathscr{K} \subset L_{b}(X, Y)$. Then $\mathscr{K}$ is a totally bounded set of semi-precompact operators if and only if

(i) $\mathscr{K}(A)$ is totally bounded for every bounded set $A$ in $X$; and

(ii) $\mathscr{K}^{*}\left(y^{*}\right)$ is totally bounded for every $y^{*}$ in $Y^{*}$.

Proof. Let $\mathscr{K}$ be totally bounded set of semi-precompact operators. Suppose that $A$ is any bounded set in $X$. Then for any 0 -neighbourhood $V$ in $Y$, there exist $K_{1}, K_{2}, \ldots, K_{m}$ in $\mathscr{K}$ such that for every $K$ in $\mathscr{K}$, there is $K_{i}$ with

$$
\left(K-K_{i}\right)(A) \subset V \text {. }
$$


Since each $K_{i}$ is semi-precompact, $\bigcup\left\{K_{i}(A): i=1,2, \ldots, m\right\}$ is totally bounded. Thus $\mathscr{K}(A)$ has a totally bounded $V$-net, namely $\bigcup\left\{K_{i}(A): i=1,2, \ldots, m\right\}$. Hence $\mathscr{K}(A)$ is totally bounded. Also for each $y^{*} \in Y^{*}, \mathscr{K}^{*}\left(y^{*}\right)=y^{*} \mathscr{K}$. Since $\mathscr{K}$ is totally bounded, $y^{*} \mathscr{K}$ is also totally bounded in $X_{s}^{*}$. Thus the 'only if' part is proved. Denote $E=x_{0} \otimes x_{0}^{*}, x_{0}^{*}\left(x_{0}\right)=1, x_{0} \in X$ and $x_{0}^{*} \in X^{*}$, and $F=y_{0} \otimes y_{0}^{*}$, $y_{0}^{*}\left(y_{0}\right)=1, y_{0} \in Y$ and $y_{0}^{*} \in Y^{*}$. To prove the converse, let (i) and (ii) hold. Let $\mathscr{K}^{\prime}$ be any infinite subset of $\mathscr{K}$ and $N(A, V)$ any 0 -neighbourhood in $L_{b}(X, Y)$. Since every locally convex topology is the topology of uniform convergence on equicontinuous subsets of its dual, there exists an equicontinuous subset $B^{*}$ of $Y^{*}$ such that $\left(B^{*}\right)^{0} \subset V$, where $\left(B^{*}\right)^{0}=\left\{y \in Y:\left|y^{*}(y)\right| \leqslant 1, y^{*} \in B^{*}\right\}$, the polar of $B^{*}$ in $Y$. Let $W_{x}=\left\{x \otimes x_{0}^{*}: x \in A\right\}, W_{y}=\left\{y_{0} \otimes y^{*}: y^{*} \in B^{*}\right\}$. Then $W_{x}, W_{y}$ are bounded subsets of $L_{b}(X), L_{b}(y)$ respectively. In addition, $W_{y}$ is equicontinuous. This can be easily deduced from the equicontinuity of $B^{*}$ in $Y^{*}$. Now for any $S$ in $L(Y)$, define $\pi_{S}: L_{b}(X, Y) \rightarrow L_{b}(X, Y)$ by the equation $\pi_{S}(T)=S T$. Let $\mathscr{F}=\left\{\pi_{F T}: F T \in F W_{y}\right\}$. From the equicontinuity of $W_{y}$, the equicontinuity of $\mathscr{F}$ follows easily. Again by (i) and Proposition 1.8, it follows that $\mathscr{K}^{\prime} W_{x} E$ is totally bounded in $L_{b}(X, Y)$. Now observe that the restriction of $\mathscr{F}$ to the totally bounded set $\mathscr{K}^{\prime} W_{x} E$ is equicontinuous and the range of this restriction is contained in the one-dimensional space generated by $y_{0} \otimes x_{0}^{*}$. Thus $\mathscr{F}$ defines an equicontinuous family of functions with values in the space of one dimension on the compact closure of $\mathscr{K}^{\prime} W_{x} E$ in the completion of $L_{b}(X, Y)$. Hence $\mathscr{F}$ restricted to $\mathscr{K}^{\prime} W_{x} E$ is totally bounded in the topology of uniform convergence on $\mathscr{K}^{\prime} W_{x} E$. Now let $p$ be a continuous semi-norm on $Y$ with $p\left(y_{0}\right)>1$ and $U=\{y \in Y: p(y) \leqslant 1\}$. Then $U$ is a 0 -neighbourhood in $Y$. Suppose $U_{1}$ is a 0 -neighbourhood in $Y$ such that $U_{1}+U_{1}+U_{1} \subset U$. Since $\mathscr{F}$ restricted to $\mathscr{K}^{\prime} W_{x} E$ is totally bounded, it follows that for $N\left(x_{0}, U_{1}\right), 0$-neighbourhood in $L_{b}(X, Y)$, there exist $T_{1}, T_{2}, \ldots, T_{m}$ in $W_{y}$ such that for each $T$ in $W_{y}$, there is $T_{i}$ with $\left(F T-F T_{i}\right) \mathscr{K}^{\prime} W_{x} E\left(x_{0}\right) \subset U_{1}$, or, equivalently, we have

$$
\left(F T-F T_{i}\right) \mathscr{K}^{\prime}(A) \subset U_{1} .
$$

Now by (ii) and Corollary $1.7, F T_{i} \mathscr{K}^{\prime}$ is totally bounded for each $T_{i}, i=1,2, \ldots, m$. Hence by Lemma 2.1, we can find for each $i$ an infinite subset $\mathscr{K}^{\prime \prime} \subset \mathscr{K}^{\prime}$ such that $F T_{i} \mathscr{K}^{\prime \prime}$ is small of order $N\left(A, U_{1}\right)$. Since the $T_{i}$ 's are finite in number, we can choose an infinite subset $\mathscr{K}^{\prime \prime} \subset \mathscr{K}^{\prime}$ such that $K_{1}, K_{2} \in \mathscr{K}^{\prime \prime}$ implies

$$
\left(F T_{i} K_{1}-F T_{i} K_{2}\right)(A) \subset U_{1} \text { for all } i=1,2, \ldots, m \text {. }
$$

Now for $K_{1}, K_{2} \in \mathscr{K}^{\prime \prime}$ and $T \in W_{y}$, we have

$$
\begin{aligned}
F T\left(K_{1}-K_{2}\right)(A) & \subset\left(F T-F T_{i}\right) K_{1}(A)+F T_{i}\left(K_{1}-K_{2}\right)(A)+\left(F T_{i}-F T\right) K_{2}(A) \\
& \subset U_{1}+U_{1}+U_{1} \text { by (a) and (b) } \\
& \subset U .
\end{aligned}
$$


Hence by definition of $U$, we have

$$
p\left[F T\left(K_{1}-K_{2}\right)(x)\right] \leqslant 1 \text { for all } x \text { in } A,
$$

or equivalently,

Hence

$$
\left|T^{*}\left(y_{0}^{*}\right)\left[\left(K_{1}-K_{2}\right)(x)\right]\right| p\left(y_{0}\right) \leqslant 1 \text { for all } x \text { in } A .
$$

$$
\left|T^{*}\left(y_{0}^{*}\right)\left[\left(K_{1}-K_{2}\right)(x)\right]\right| \leqslant 1 / p\left(y_{0}\right)<1 \text { for all } x \text { in } A .
$$

Since $W_{y}^{*}\left(y_{0}^{*}\right)=B^{*}$, we have

$$
\left|y^{*}\left[\left(K_{1}-K_{2}\right)(x)\right]\right|<1 \text { for all } x \text { in } A
$$

and $y^{*} \in B^{*}$. Thus we have $\left(K_{1}-K_{2}\right)(A) \subset\left(B^{*}\right)^{0} \subset V$. This proves that $\mathscr{K}^{\prime \prime}$ is an infinite subset of $\mathscr{K}^{\prime}$ which is small of order $N(A, V)$. This proves the total boundedness of $\mathscr{K}$ in $L_{b}(X, Y)$.

Corollary 2.3. Let $X, Y$ be locally convex Hausdorff spaces and $\mathscr{K} \subset L_{b}(X, Y)$. If $\mathscr{K}$ and $\mathscr{K}^{*}$ are collectively precompact, then $\mathscr{K}$ is totally bounded.

The converse of this corollary is not true. A counter example to show this was constructed by DePree and Higgins (1970). But their example is quite complicated. Here we give a much simpler example.

EXAMPLE 2.4. Let $X=\left(l^{2}, w\right)$, that is the space $l^{2}$ with weak topology. By definition, the weak topology on $l^{2}$ is the topology generated by points of $l^{2}$ through inner product of $l^{2}$. Let $e_{n}$ denote the vector which is 1 in the $n$th coordinate and 0 in all others. For $x$ in $l^{2}$, let $x_{n}$ denote the $n$th coordinate of $x$. Define $P_{n}(x)=x_{n} e_{n}$ for each $n \geqslant 1$. Then we show the following:

(i) Each $P_{n}$ is a precompact operator.

(ii) $P_{n} \rightarrow 0$ in $L_{b}(X)$.

(iii) $\left\{P_{n}: n \geqslant 1\right\}$ is not collectively precompact.

Proof. (i) It is easy to check that each $P_{n}$ is continuous. Since $P_{n}$ 's are one rank operators, they are all precompact operators.

(ii) Let $N(B, U)$ be any 0 -neighbourhood in $L_{b}(X)$, where $U$ is the polar of some finite set in $X$, say $\left\{y_{i}: i=1,2, \ldots, m\right\}$. Since $B$ is bounded,

$$
\sup \left\{\left|\left(x, e_{n}\right)\right|: x \in B, n \geqslant 1\right\} \leqslant M \text {. }
$$

Also $\left(e_{n}, y_{i}\right) \rightarrow 0$ for every $i$. Hence, we can find a positive integer $N_{0}$ such that $\left|\left(e_{n}, y_{i}\right)\right| \leqslant 1 / M$ for all $n \geqslant N_{0}$ and $i=1,2, \ldots, m$. Therefore, for any $x$ in $B$ and $n \geqslant N_{0}, \quad\left|\left(P_{n}(x), y_{i}\right)\right|=\left|x_{n} \|\left(e_{n}, y_{i}\right)\right| \leqslant M(1 / M)=1$. Thus $P_{n} \in N(B, U)$ for all $n \geqslant N_{0}$.

(iii) Let $U$ be any 0 -neighbourhood in $X$. Then $U$ is the polar of some finite set 
in $X$, say $\left\{z_{i}: i=1,2, \ldots, p\right\}$. Choose $x$ in $X$ and $n \geqslant 1$ such that $\left(x, z_{i}\right)=0$ for all $i=1,2, \ldots, p$ and $\left(x, e_{n}\right) \neq 0$. Since $\{m x: m \geqslant 1\} \subset U$ and $P_{n}(x) \neq 0$, the set $P_{n}\{m x: m \geqslant 1\}$ is unbounded subset of $\bigcup\left\{P_{i}(U): i \geqslant 1\right\}$. Hence $\bigcup\left\{P_{i}(U): i \geqslant 1\right\}$ is unbounded and therefore not totally bounded.

Proposition 2.5. Let $X$ be infra-barrelled space (for definition, see Horvath (1966)) and $T \in L(X)$. Then the map $K \rightarrow T K T$ from $L_{b}(X)$ into itself is semi-precompact if and only if $T$ is semi-precompact operator.

Proof. Let $T$ be semi-precompact and $W$ be any bounded subset of $L_{b}(X)$. To prove the total boundedness of $T W T$, we use Theorem 2.2. But for every bounded set $A$ of $X, T W T(A)$ is obviously totally bounded in $X$. Since $X$ is infra-barrelled $T^{*}$ is also semi-precompact. Also boundedness of $W$ implies pointwise boundedness of $W^{*}$. This proves the total boundedness of $T^{*} W^{*} T^{*}\left(x^{*}\right)$ for every $x^{*}$ in $X^{*}$. Hence $T W T$ is totally bounded. Conversely, let $T W T$ be totally bounded for every bounded set $W$ in $L_{b}(X)$. To prove semi-precompactness of $T$, let $B$ be any bounded subset of $X$. Then $W=\left\{x \otimes x_{1}^{*}: x \in B\right\}$ is bounded in $L_{b}(X)$, where $x_{1}^{*} \in X^{*}$ such that $x_{1}^{*}\left[T\left(x_{1}\right)\right]=1$ for some $x_{1}$ in $X$. But then $T(B)=T W T\left(x_{1}\right)$ is totally bounded in $X$.

\section{References}

P. M. Anselone (1970), 'Compactness properties of sets of operators and their adjoints', Math. $Z$. 113, 233-236.

J. D. DePree and J. A. Higgins (1970), 'Collectively compact sets of linear operators', Math. $Z$. 115, 366-370.

J. D. DePree and H. S. Klein (1974), 'Characterizations of collectively compact sets of linear operators', Pacific J. Math. 55, 45-54.

A. S. Geue (1974), 'Precompact and collectively semi-precompact sets of semi-precompact continuous linear operators', Pacific J. Math. 52, 377-401.

J. Horvath (1966), Topological vector spaces and distributions (Addison-Wesley, Reading, Massachusetts).

T. W. Palmer (1969), 'Totally bounded sets of precompact linear operators', Proc. Amer. Math. Soc. 20, 101-106.

Department of Mathematics Indian Institute of Technology Powai, Bombay 400076

India 